\title{
Stereo Vision Distance Estimation Employing SAD with Canny Edge Detector
}

\author{
Raad H. Thaher \\ College of Engineering \\ Al-Mustansiriyah University \\ Baghdad /Iraq
}

\author{
Zaid K. Hussein \\ College of Engineering \\ Al-Mustansiriyah University
}

\begin{abstract}
Stereo vision system used to reconstruct a 3D scene from 2D images taken by a pair of optical cameras (left and right images) and it is used to estimate the distance of the object.

The modified version for the (SAD) algorithm is called the Canny Block Matching Algorithm (CBMA) to find the Disparity map, the algorithm consist of two parts the Canny edge detector and Block matching technique with Sum of Absolute Difference (SAD) to determine disparity map to reduce the execution time. The system has been implemented using two cameras arranged in a manner to enhance the detection range of objects from $(30 \mathrm{~cm}$ to $4 \mathrm{~m})$. The results show good outputs with less error percentage, as compared with the real objects depth and the Execution time is approximated to the real-time performance. The algorithms implemented using MATLAB (8.0) technical programing language.
\end{abstract}

\section{Keywords}

Stereo Vision, Disparity, Epipolar geometry, SAD, CBMA.

\section{INTRODUCTION}

Stereoscopic vision is a technique for inferring the $3 \mathrm{D}$ position of objects from two or more simultaneous images of a scene. Mobile robots can take advantage of a stereo vision system as a reliable and effective way to extract 3D data from the environment. [1]

Reconstruction of the world seen (3D information) by stereo vision cameras can be divided into two steps:

1-Correspondence problem: For each point in image and the corresponding point in the other image and compute the disparity distance in pixels of these points.

2-Triangulation: Given the disparity map and focal distance of the two cameras and the geometry of the stereo setting relative position and orientation of the cameras compute the world coordinates of each point in the images [1].

The depth is obtained from computing stereo matching between two images. The technique is triangulation, which represents the process of finding coordinates of a 3D point based on its corresponding stereo image points [2].

A number of studies have been reported on the disparity estimation problem since 1970's like Marr-Poggio In 1976 and 1979, [3][4]they first work in stereo vision system and used a cooperative algorithm to extracting disparity information from stereo image pairs.. Many researches in the literature deals with scope of this work, as "Eric" and "Grimson" in 1984. [5], "Hakkarainen" and "Lee", In 1993. [6], "Labayirade" and "Aubert" In 2002. [7],"Vatansever "In 2006. [8], and "Fengjun HU" and "Yanwei ZHAO" in 2013 [9].
Extracting depth map from stereo image pairs is achieved by applying matching algorithm, which is based on two criteria that represent the main challenges of stereo vision system, the main criteria are:

1. The execution time: the execution of algorithm should be fast.

2. Reliability: the information about objects should be accurate enough to recognize.

\section{STEREO VISION SYSTEM}

The term stereo vision refers to the theory that is two cameras properly configured can be used to map objects within a $2 \mathrm{D}$ scene into three $3 \mathrm{D}$ spaces.

The computer compares the images while shifting the two images together over top of each other to find the parts that match or are similar, and the shifted amount is called (disparity) that is related to the object distance the higher disparity of the object pixel means that the object is closer to the cameras and the less disparity means the object is far from the cameras [10] [11]. Disparity maps can be visualized in greyscale. Close objects result in a large disparity value. This is translated into light greyscale values. Objects further away will appear darker.

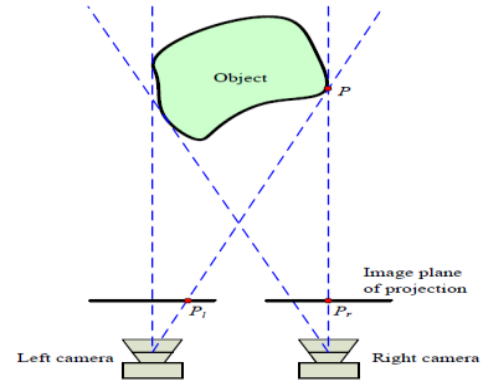

Fig. (1): the positions of two cameras and their image planes of projection

Figure (1) shows the geometrical basis for stereoscopic image with two identical cameras. The two cameras are set up on the same plane (parallax sight). The position of both cameras is different in $\mathrm{X}$ axis, and the image planes are presented in front of the cameras for ease to model the projection [11]

\section{STEREO CAMERA GEOMETRY}

The epipolar geometry of stereoscopic camera is shown in Figure (2) this is stereo model shows two different perspective views of an object point (p) from two cameras centers $\left(F_{l}\right.$ and $\left.F_{r}\right)$. The points $p_{l}$ and $p_{r}$ in the image plane are the perspective projections of $(\mathrm{P})$ in left and right view, called a conjugate pair. The plane passes through the camera centers and the object point in the scene is called the 
epipolar plane, correspondences at point $p_{l}$ and $p_{r}$ must lie on the epipolar line.

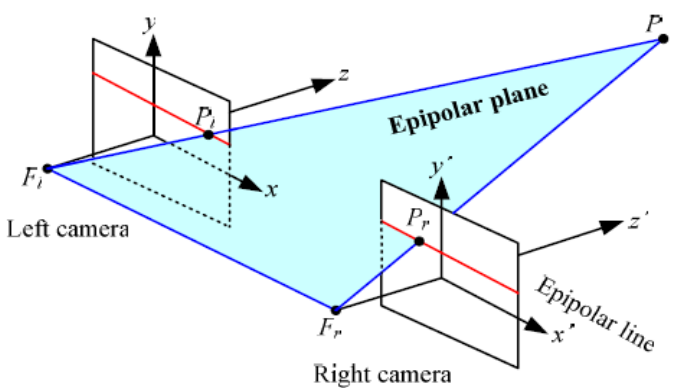

Fig. (2): The epipolar geometry of stereoscopic vision.

The simplest stereo camera geometry has optical axes that is parallel and is normal to the baseline; that line is connected to the lens centers of the cameras.

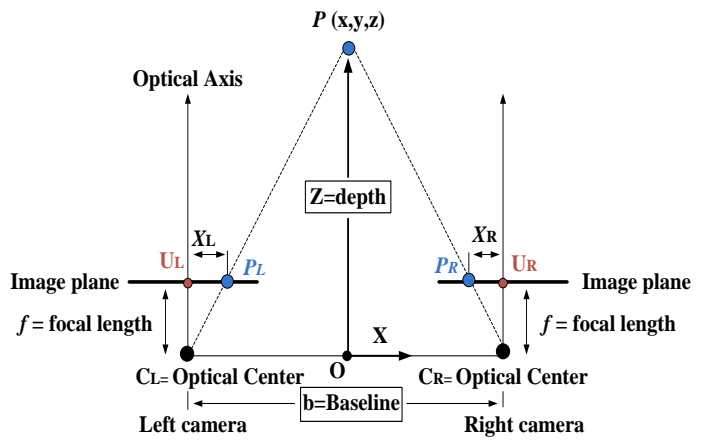

Fig. (3): Epipolar geometry with parallel optical axes (top view)

As shown in Figure (3) the stereo camera coordinate system considered to be midway between the left and right camera coordinate systems. Assuming idealistic (pin-hole) cameras, so the stereo camera coordinate system can be written, from similar triangles in Figure (3), for the left and right camera we have:

$$
z=\frac{b * f}{x_{L}-x_{R}}=\frac{b * f}{d}
$$

The quantity $\left(\mathrm{d}=x_{L}-x_{R}\right)$ is called the disparity, (b) is the base line and (f) is the focal length of the cameras. The Relation (1) indicates that the depth is inversely proportional to disparity. The larger disparity value is closer to the object point to the camera base line and the smaller disparity value is the more distant to the object point from the camera baseline, and for the same depth a larger disparity is obtained when a camera system with a larger focal length or a larger baseline length is used [12].

\section{SUM OF ABSOLUTE DIFFERENCE ALGORITHM (SAD)}

Sum of Absolute Difference (SAD) technique is the most common matching criteria in stereo matching algorithms cause by its low complexity, good performance and ease of hardware implementation.

The (SAD) calculates the differences between two selected windows the search is done by moving the reference window pixel-by-pixel (full search) over the target windows that minimizes the associated error, the SAD equation is [13]:

$$
\mathrm{SAD}=\sum_{(\mathrm{i}, \mathrm{j}) \in W(\mathrm{x}, \mathrm{y})}\left|\mathrm{I}_{\mathrm{L}}(\mathrm{i}, \mathrm{j})-\mathrm{I}_{\mathrm{R}}(\mathrm{i}, \mathrm{j}+\mathrm{d})\right|
$$

Where $I_{L}$ and $I_{R}$ are the intensity for the pixel of the left and right image, respectively. $\mathrm{W}(\mathrm{x}, \mathrm{y})$ is square window that surrounds the location ( $\mathrm{x}, \mathrm{y})$ for the pixel. And $\mathrm{d}$ is the disparity value. In order to implement a reliable algorithm and to demonstrate its effectiveness, it has been tested with many real and rectified stereo image pairs, provided as database [14] with ground-truth (true disparity map) that is required to calculate the percentage error. One of the stereo pairs called (Tsukuba) with size of $(384 \times 288)$, as shown in Figure (4).

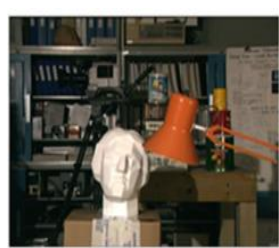

(a) Left image

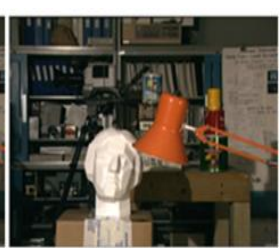

(b) Right image

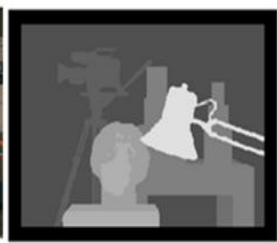

(c) true disparity map
Fig. (4): Tsukuba stereo image pair and true disparity map.

To discuss the results of the matching algorithm, it has been executed at different window sizes to illustrate the effect of window size on the execution time. Figure (5) shows the results of disparity maps for Tsukuba stereo image pair test by SAD algorithm for different sizes of local support windows $([3 \times 3],[7 \times 7],[11 \times 11],[15 \times 15],[19 \times 19]$ and $[25 \times 25])$.

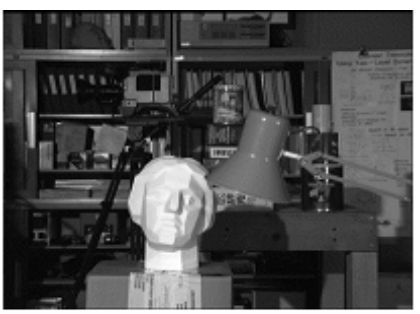

(a) Gray scale image

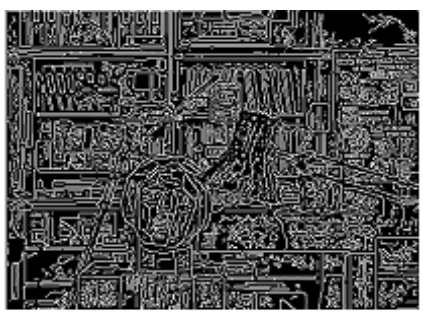

(b) Filtered image at thigh $=0.01$

$$
\& \sigma=0.5
$$

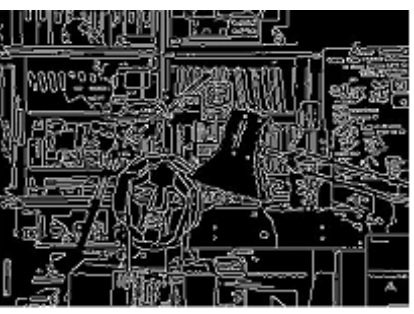

(c) Filtered image at thigh $=0.05$ $\& \sigma=1$

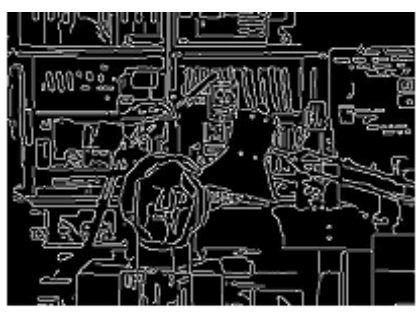

(d) Filtered image at thigh $=0.1$ $\& \sigma=1$

Fig. (5): Disparity maps of Tsukuba SAD algorithm for different window sizes. 
Table (1): The execution time and error calculationaccording to SAD

\begin{tabular}{|l|l|l|}
\hline $\begin{array}{l}\text { Window- } \\
\text { size }\end{array}$ & $\begin{array}{l}\text { Error percentage } \\
(\%)\end{array}$ & $\begin{array}{l}\text { Execution time } \\
(\text { Sec. })\end{array}$ \\
\hline$[3 \times 3]$ & 23.7957 & 1.1540 \\
\hline$[5 \times 5]$ & 17.4613 & 2.3169 \\
\hline$[7 \times 7]$ & 15.2788 & 4.0723 \\
\hline$[9 \times 9]$ & 12.3796 & 6.2336 \\
\hline$[11 \times 11]$ & 11.3608 & 8.7930 \\
\hline$[13 \times 13]$ & 11.5203 & 11.8933 \\
\hline$[15 \times 15]$ & 10.9002 & 15.4039 \\
\hline$[17 \times 17]$ & 10.9211 & 19.2220 \\
\hline$[19 \times 19]$ & 10.5556 & 23.5235 \\
\hline$[21 \times 21]$ & 11.4709 & 27.9363 \\
\hline$[23 \times 23]$ & 10.7036 & 33.3579 \\
\hline$[25 \times 25]$ & 11.6620 & 38.4058 \\
\hline$[27 \times 27]$ & 10.9185 & 44.2607 \\
\hline
\end{tabular}

From Figure (5), and the results of shown in Table (1), It can be noticed that, the optimal window size is approximately from [19×19] since it gives less error percentage $(10.5556 \%)$ and execution time is $(23.5235 \mathrm{sec})$, but when we take the near value of the less error percentage is $(10.9002 \%)$ at window size is [15x15] and execution time is (15.4039 sec) the time is less than that in the window size is [19x19]. The result of stereo vision algorithm is a grayscale disparity map which indicates the disparity for each pixel with corresponding intensity. The lighter areas in Figure (5) are closer to the camera, darker ones further away.

\section{CANNY BLOCK MATCHING ALGORITHM (CBMA)}

The algorithm is consists of two stages from first stage a Canny edge detector and the second stage is Block matching technique using SAD for determined the disparity map.

\subsection{Canny Edge Detector}

The Canny edge detector is an edge detection operator which is using a multi-stage algorithm to detect a wide range of edges in the images. The Canny edge detector uses the first derivative of a Gaussian; so to begin with, the raw image is convolved with a Gaussian filter. The result is a slightly blurred version of the original, and the edge direction angle is rounded to one of four angles representing vertical, horizontal and the two diagonals. [15][16]

Canny uses thresholding with hysteresis, and they require two thresholds (high and low). And making the assumption that is the important edge should be along continuous curves in the image allows to follow a faint section of a given line and to discard a few noisy pixels that do not constitute a line but have produced large gradients; therefore, begin by applying a high threshold.[17]

The Canny operator has three variables (Sigma standard deviation of Gaussian $(\sigma)$ and two thresholds (tlow and thigh)): sigma range from (0.5 - 5), thigh range from (0.010.2 ), and tlow is depending on thigh as tlwo $=0.4 \mathrm{xthigh}$.

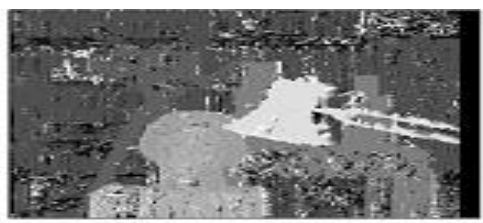

(a) $[3 \times 3]$

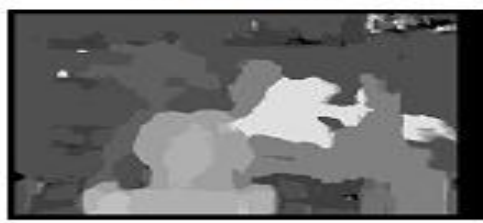

(d) $[15 \times 15]$

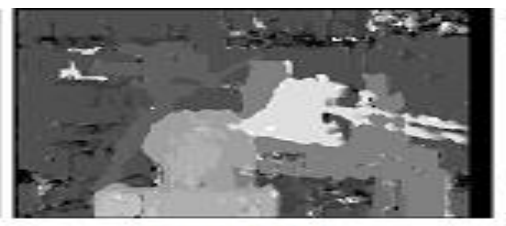

(b) $[7 \times 7]$

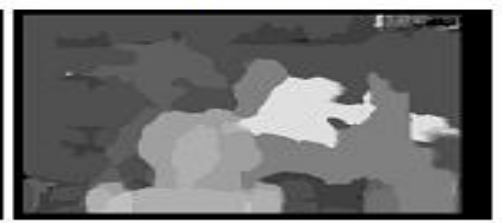

(e) $[19 \times 19]$

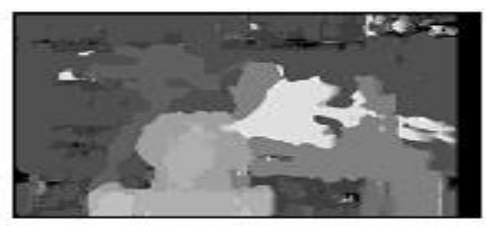

(c) $[11 \times 11]$

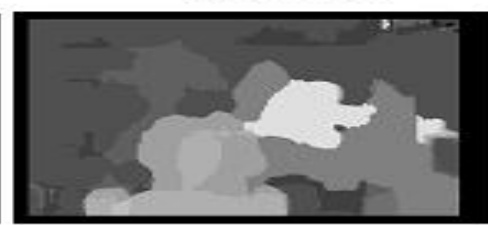

(f) $[25 \times 25]$

Fig. (6): Tsukuba image, (a) before applying Canny filter. And after applying Canny edge detector at different threshold and sigma values: (b) at thigh $=0.01 \& \sigma=0.5$, (c) at thigh $=0.05 \& \sigma=1$ and (d) at thigh $=0.1 \& \sigma=1$.

The optimal result for this image is obtained at threshold value of in ((c) (tlow $=0.4 \mathrm{xthigh}$, thigh $=0.05 \& \sigma=1$ and the time is 0.174785 seconds)), it can be considered as an optimal value since it compromises between accuracy of matching results and speed up the execution time.

\subsection{Block Matching Techniques}

Block matching is a technique in stereo vision, which is used to solve the correspondence problem, every block from the left image, is matched into a block in the right image by shifting the left block over the searching area for pixels in right image, as shown in Figure (7) at each shift. [18]

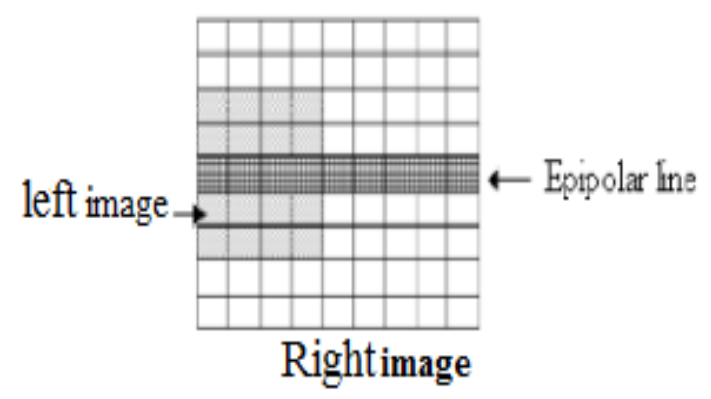

Fig. (7): SAD block matching techniques 
In order to reduce the computational time, in which the search for the matched blocks is performed by moving the reference window of the reference image block-by-block over the target image, and the disparity value calculated for the central pixel of the window represents the same disparity value for all window's pixels neighboring the central pixel. By this method large amount of SAD computation time can be reduced.

\subsection{Algorithm Steps}

The CBMA algorithm can be summarized as several major steps, as follows:

Step1. Read the two real and rectified images (right image and left image).

Step2. Convert both images from (RGB scale) color image to Gray scale image.

Step3. Set the value of Standard deviation of Gaussian $(\sigma=1)$

Step4. Set the values of Canny edge detector thresholds (thigh=0.05) and tlow=0.4thigh.

Step5. Set the value of "Search Range" $(d=0, \ldots, d \operatorname{dmax}=16)$ that represents the search range within a loop.

Step6.Set the size of the two matching window (block) for search rows and columns.

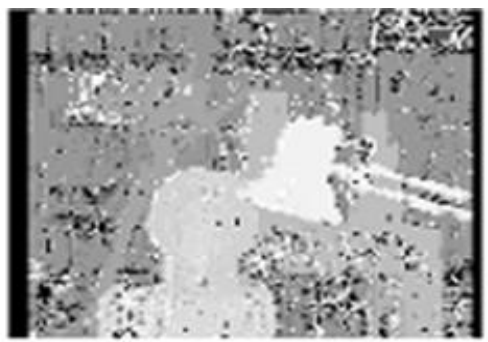

(a) $[3 \times 3]$

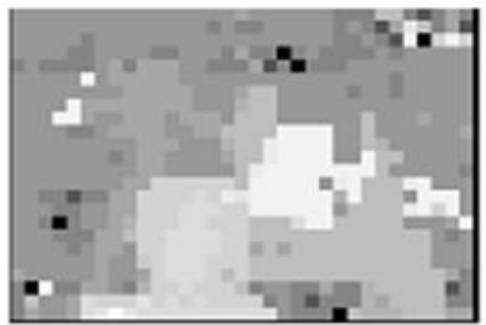

(d) $[15 \times 15]$

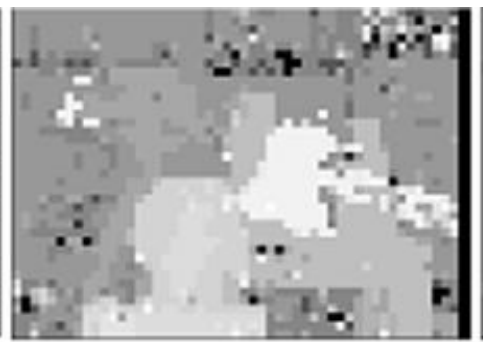

(b) $[7 \times 7]$

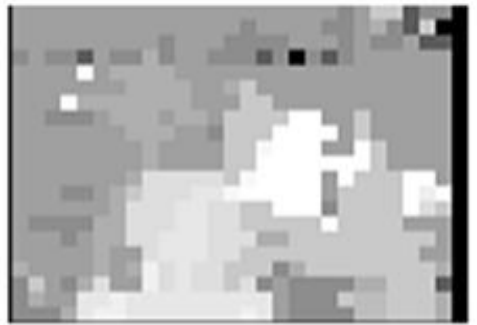

(e)[19x19]
Step7. Prepare a 3D array (x, y, d) for disparity map.

Step8. Set initial match value (Initial SAD) using a function of image intensities calculated by sum of absolute differences

Initial SAD $=2^{\wedge} 16-1=65535$

Step9. Compute SAD between reference window and candidate windows at each shift (value of dmax) according to the Equation (2).

Step10. Iteratively update the initial SAD value with every new minimum SAD computed, until the match value converges. Note that the matching is computed for minimum $\mathrm{SAD}$ value since; it represents the mean error value.

Step11. For each pixel $(x, y)$ of the reference image, find the disparity element $(\mathrm{x}, \mathrm{y}, \mathrm{d})$, where the disparity value at which minimum SAD is obtained. The set of disparity values represents the disparity map.

After testing the CBMA algorithm with the pair's images for (Tsukuba) the results for the disparity map are obtained in Figure (8), the execution time and error calculation according to CBMA algorithm are in the Table (2).

Fig. (8): Disparity maps of Tsukuba image result from CBMA for different window sizes.

Table (2): The execution time and error calculation according to CBMA

\begin{tabular}{|l|l|l|}
\hline Window-size & $\begin{array}{l}\text { Error percentage } \\
(\%)\end{array}$ & $\begin{array}{l}\text { Execution time } \\
(\text { Sec. })\end{array}$ \\
\hline$[3 \times 3]$ & 23.87 & 0.4379 \\
\hline$[5 \times 5]$ & 18.03 & 0.3771 \\
\hline$[7 \times 7]$ & 14.91 & 0.3984 \\
\hline$[9 \times 9]$ & 13.56 & 0.3657 \\
\hline
\end{tabular}

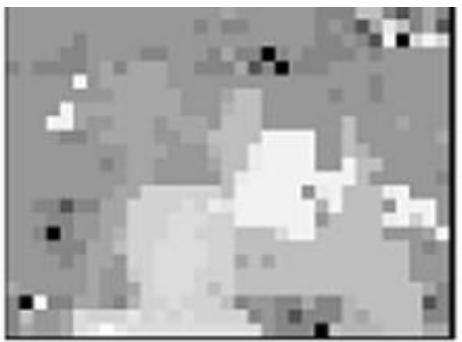

(c) $[11 \mathrm{x} 11]$

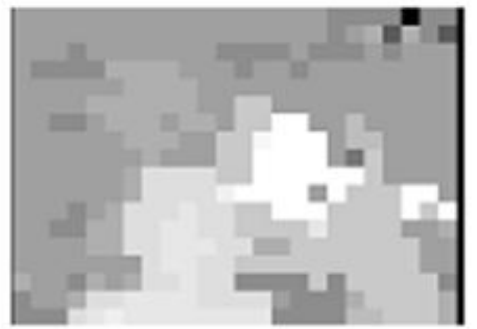

(f) $[25 \times 25]$

\begin{tabular}{|l|l|l|}
\hline$[11 \times 11]$ & 12.68 & 0.3593 \\
\hline$[13 \times 13]$ & 11.24 & 0.3718 \\
\hline$[15 \times 15]$ & 12.93 & 0.3689 \\
\hline$[17 \times 17]$ & 13.20 & 0.3611 \\
\hline$[19 \times 19]$ & 13.40 & 0.3685 \\
\hline$[21 \times 21]$ & 13.11 & 0.3709 \\
\hline$[23 \times 23]$ & 14.22 & 0.3728 \\
\hline$[25 \times 25]$ & 14.47 & 0.3639 \\
\hline
\end{tabular}




$$
\text { [27×27] }
$$

$$
13.40
$$

\subsection{5}

From Table (2) by this method, large amount of SAD computation can be reduced, consequently the computation time is dramatically reduced as shown the execution time between $(0.4379$ and 0.3593$) \mathrm{sec}$ is good for application to robots. The Error percentage with different windows shows the optimal window as [13x13] and gives the less error percentage with execution time which is $(0.3718) \mathrm{sec}$. So, the algorithm (CBMA) has reduced the execution time, but the Error percentage is slightly larger than the other algorithms, but the window [13x13] is taken good to do the work to compute the disparity map.

\subsection{Comparison of the Results}

The error percentage and the execution time results from the (SAD) and (CBMA) algorithms are carried out for Tsukuba image pair is compared. So, the values of the error percentage and the values of the execution time are taken from Tables (1) and (2) and used in Figures (9) and (10) respectively.

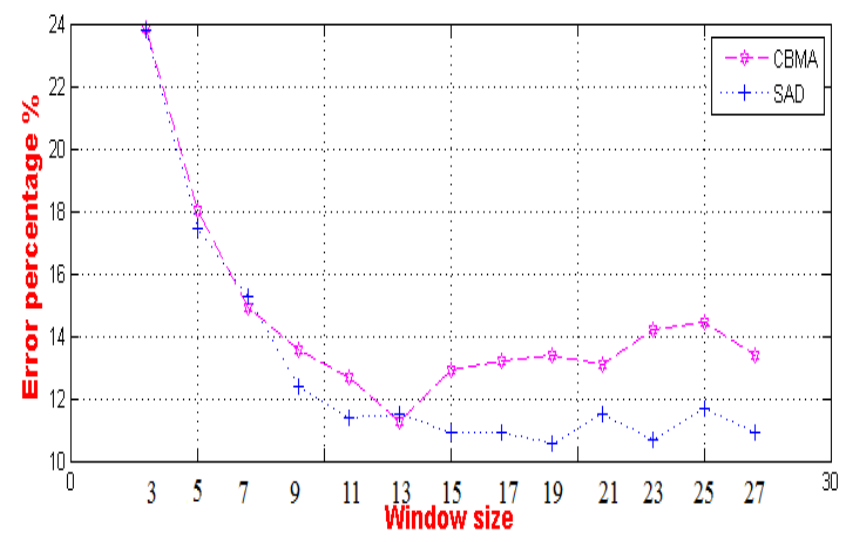

Fig. (9): Compare Results Error percentage between SAD \& CBMA

As shown in Figure (9), the Error percentage of SAD is slightly better than the CBMA about (2\%), the smallest value of the error percentage in SAD is $(10.55 \%)$ with execution time $(23.523 \mathrm{sec})$ at window which is [19x19] and the smallest value of the error for CBMA is $(11.24 \%)$ with execution time $(0.372 \mathrm{sec})$ at window [ $13 \times 13]$ so the advantage can be taken for the smallest execution time achieve with the CBMA.

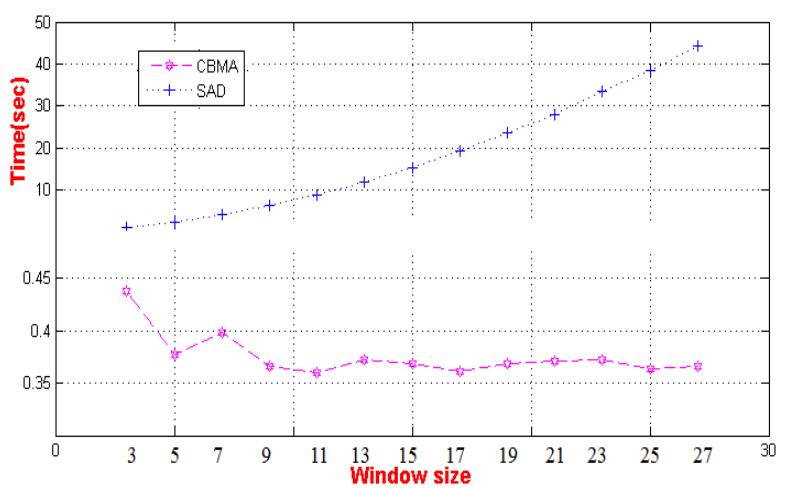

Fig. (10): Comparison Results Execution time between SAD \& CBMA

As shown in Figure (10) the large amount of reduction in execution time for CBMA has been achieved with different image pairs. This reduction is attributed to the search for the matched blocks technique (Block-by-Block) instead of (pixelby-pixel) search technique used in SAD algorithm. The reduction CBMA algorithm between (0.4379 - 0.3593) sec is very large, and the relation between the window size and the execution in the SAD algorithm is removed when the window size large and the execution time are large too, but in CBMA algorithm, the window size effect the error percentage only and no big change on the execution time.

\section{STEREO VISION HARDWARE}

The vision system hardware used is composed of two identical models USB web cameras connected to PC with USB cable, as shown in Figure(11).The camera specification, High Resolution CMOS Color Sensor, Resolution: 1300k pixel (interpolated $5 \mathrm{M}$ ), Interface: 2.0, Photo quality of 3 Megapixels. The system operates at a resolution of $320 \times 240$. This is generally suitable for vision system and actually reduces the number of pixels that requires more computation time. The selected value of baseline enables to a working area of stereo vision (FOV) $4.45 \mathrm{~m}$ width at range of $4 \mathrm{~m}$.

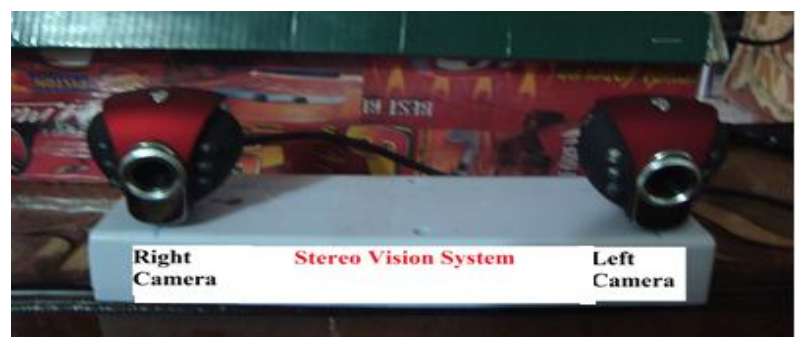

Fig. (11): The implemented stereo vision system

\section{DEPTH MEASUREMENT}

After stereo cameras have been calibrated and their parameters (intrinsic and Extrinsic) have been calculated, and rectification the images from that parameters the depth of the scene objects can be extracted. The correspondence algorithm has been applied to extract disparity map.

In order to calculate the accuracy of the estimated depth $z$, a comparison has been made between the estimated depth values and the real depths.

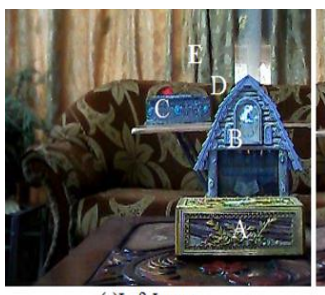

(a)Left Image

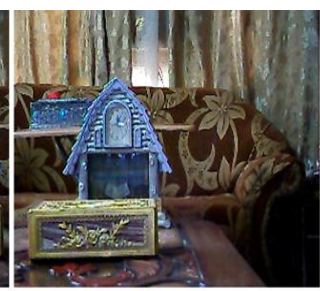

(b)Right Image

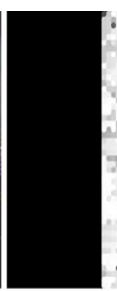

Fig. (12): Selecting different points at different depths, shows stereo images captured from the implemented stereo cameras, and the disparity map.

Table (3): Distance measurements of the five points of Figure (12)

\begin{tabular}{|l|l|l|l|l|}
\hline $\begin{array}{l}\text { Tested } \\
\text { points }\end{array}$ & $\begin{array}{l}\text { disparity } \\
\text { value (d) in } \\
\text { (pixels) }\end{array}$ & $\begin{array}{l}\text { Real depth } \\
(\mathrm{cm})\end{array}$ & $\begin{array}{l}\text { Estimated depth } \\
(\mathrm{cm})\end{array}$ & $\begin{array}{l}\text { Depth Error } \\
\text { percentage } \\
(\%)\end{array}$ \\
\hline $\mathrm{A}$ & $\mathbf{7 7}$ & 121 & 121.887 & $+\mathbf{0 . 7 2} \%$ \\
\hline
\end{tabular}




\begin{tabular}{|l|l|c|c|c|}
\hline B & 59 & 160 & 159.02 & $-0.6 \%$ \\
\hline C & 40 & 236 & 234.59 & $-\mathbf{0 . 5 9} \%$ \\
\hline D & 31 & 297 & 302.7 & $+1.9 \%$ \\
\hline E & 29 & 313 & 323.58 & $+3.38 \%$ \\
\hline
\end{tabular}

The estimated depths of the randomly selected points in the real scene are calculated according to the depth equation, in which the focal length (f) that represents one of the internal parameters of the camera is extracted from camera calibration as mentioned before, where the horizontal focal length in pixels of the left camera is $\alpha=586.491$ pixels

At points $\mathrm{A}, \mathrm{B}$ and $\mathrm{C}$, less error in the depth results is obtained, since the disparity values obtained at these points are correct. And for points D and E the error is begin to by bigger because the values of the disparity is in correct.

From Table (3) it can be also noted that, the disparity value decreases for the objects which are far from the camera and the object appears darker and vice versa. For example, the closest point to the cameras (point A) at which the object appears lighter and it has large disparity value of (77 pixels), while at point $\mathrm{D}$ small disparity value is obtained and the objects appear darker.

\section{CONCLUSIONS}

The main important conclusions can be summarized:

The execution time of the algorithm (CBMA) is approximate the real-time performance caused by using Canny filter has a noticeable effect on enhancing the reliability of the disparity map in addition to reduce the time required to execute this algorithm, and using Block Matching Techniques searching technique has a great influence on reducing the computational time operation required to calculate SAD for disparity extraction.

The selection of window size does not only influence the execution time of the algorithm, but also the accuracy of disparity map. The results show good outputs with less error, as compared with the real objects depth, which is a good result of disparity (d) values obtained by the CBMA, The selection of baseline $(16 \mathrm{~cm})$ has a direct effect on specifying the detection depth for the vision system and on the calculated depth accuracy.

\section{REFERENCES}

[1] Iocchi L. and Konolige K., "A Multiresolution Stereo Vision System for Mobile Robots", Proc. of Al Workshop on New Trends in Robotics, 1998.

[2] G. Macesanu, S. Grigorescu , T.T. Cocias , and F. Mol doveanu, "An Object Detection and 3D Reconstruction Approach For Real-Time Scene Understanding " Bulletin of the Transylvania University of Brasov, Series I: Engineering Sciences • Vol. 4 (53) No. 1 - 2011.
[3] Marr D., and Poggio T., "Cooperative Computation of Stereo Disparity", Science, New Series, Vol. 194, No. 4262, pp. 283-287, Oct. 15, 1976.

[4] Marr D., and Poggio T., "A Computational Theory of Human Stereo Vision", proceedings of the Royal Society of London. Series B, Biological Sciences, Vol. 20, No. 1156, pp. 301-328, May 23, 1979.

[5] Eric W., and Grimson L., "Computational Experiments with a Feature Based Stereo Algorithm", Massachusetts Institute of Technology, Jan., 1984.

[6] Hakkarainen J., and Lee, "A 40×40 CCD/CMOS Absolute-Value-of-Difference Processor for Use in a Stereo Vision System", IEEE, Journal of Solid-State Circuits, Vol. 28, No. 7, pp. 799-807, July 1993.

[7] Labayrad R. and Aubert D. "Robust and Fast Stereovision Based Road Obstacles Detection for Driving Safety Assistance", IAPR Workshop on Machine Vision Application, pp. 624-627, Japan, Dec., 2002.

[8] Vatansever M., "3D Reconstruction Using a Spherical Spiral Scan Camera", M.Sc. Thesis, Computer Engineering, Izmir Institute of Technology, Izmir, 2006.

[9] Fengjun HU and Yanwei Zhao "Comparative Research of Matching Algorithms for Stereo Vision ", Journal of Computational Information Systems 9: 13, PP 54575465, 2013.

[10] Adam Tawfik Sharkasi, "Stereo Vision Based Aerial Mapping Using GPS and Inertial Sensors ", Virginia Polytechnic Institute and State University, Thesis, M.Sc. April 30, 2008.

[11]Chinapirom T., Witkowski U., and Ruckert U., "Stereoscopic Camera for Autonomous Mini-Robots Applied in KheperaSot League", Heinz Nixdorf Institute, University of Paderborn, Germany, 2007.

[12] Goshtasby A., "2-D and 3-D Image Registration", a John Wiley \& Sons, Inc., Book, 2005.

[13] Myron Z. Brown, "Advances in Computational Stereo", IEEE Transactions on Pattern Analysis and Machine Intelligence, VOL. 25, NO. 8, August, 2003.

[14] http://vision.middlebury.edu/stereo/

[15] Raman Maini and Dr. Himanshu Aggarwal,"Study and Comparison of Various Image Edge Detection Techniques", International Journal of Image Processing (IJIP), Volume (3): Issue (1), pp. 1-12 2001.

[16] http://en.wikipedia.org/wiki/Canny_edge_detector

[17] https://kobefyp.googlecode.com/hg/doc/Midterm\%20Pro gress\%20Report.docx.

[18] Adam Tawfik Sharkasi, "Stereo Vision Based Aerial Mapping Using GPS and Inertial Sensors ", Virginia Polytechnic Institute and State University, Thesis, M.Sc. April 30, 2008. 